\title{
Anthropometric Indices Based on Waist Circumference as Measures of Adiposity in Children
}

\author{
Susana Santos $\mathbb{1}^{1}$, Milton Severo ${ }^{1,2}$, Carla Lopes ${ }^{1,2}$, and Andreia Oliveira ${ }^{1,2}$
}

Objective: The aim of this study was to compare the accuracy of body fat patterns and single measures in assessing body fat and to clarify the use of indices based on waist circumference as measures of adiposity in children.

Methods: This study included 2,531 7-year-old children from the Generation XXI birth cohort (Porto, Portugal). Anthropometrics were obtained by trained personnel and whole-body dual-energy $\mathrm{x}$-ray absorptiometry (DXA) scans were performed.

Results: Principal component analysis identified two body fat patterns. Pattern 1 (BMI, fat mass index from tetra-polar bioelectric impedance, and waist-to-height ratio) showed strong correlations with DXA fat mass index $(r=0.85 ; P<0.001)$, and pattern 2 (waist-to-hip, waist-to-thigh, and waist-to-weight ratios) showed moderate correlations with DXA central fat $(r=0.35 ; P<0.001)$. Compared with single measures, body fat patterns showed similar correlations with DXA-derived measures.

Conclusions: Compared with single anthropometric indices, body fat patterns seem to add little value for estimating body fat in children. Waist-to-height ratio seems to be a proxy for total fat, while waist-tohip, waist-to-thigh, and waist-to-weight ratios seem to be proxies for central fat. Further studies should address the predictive ability of waist-to-weight ratio to identify children at risk of developing cardiometabolic disease, as this was the first time describing its potential usefulness.

Obesity (2018) 26, 810-813. doi:10.1002/oby.22170

\section{Introduction}

Studies on childhood obesity have often used proxies and single measures for its definition, which might have limited their ability to detect associations (1). The combination of anthropometric indices into robust body fat patterns could be a more accurate and yet simple approach to evaluate childhood obesity. However, no study has addressed whether body fat patterns are more accurate than single measures for assessing body fat in children. Another drawback of most previous studies is related to the use of waist circumference (WC) as a proxy for central fat. WC is more strongly correlated with BMI and total body fat than with abdominal visceral fat and thus should be considered a marker of total fat $(2,3)$. This evidence raises doubts about the use of indices based on WC, such as waist- to-height ratio, as proxies for central fat, but no study has addressed this to date.

We aimed to compare the accuracy of body fat patterns and single measures for assessing body fat and to clarify the use of indices based on WC as measures of adiposity in children.

\section{Methods}

This study included participants from the population-based birth cohort Generation XXI (Porto, Portugal) (4). Of 5,849 children who attended the face-to-face interviews at 7 years old, 5,719 children had anthropometrics and tetrapolar bioelectric impedance available.

\footnotetext{
${ }^{1}$ EPIUnit, Institute of Public Health, University of Porto, Rua das Taipas, Porto, Portugal. Correspondence: Andreia Oliveira (acmatos@ispup.up.pt) ${ }^{2}$ Department of Public Health and Forensic Sciences and Medical Education, Unit of Epidemiology, Faculty of Medicine, University of Porto, Porto, Portugal.
}

Funding agencies: Generation XXI was funded by FEDER through the Operational Programme Competitiveness and Internationalization and with national funding from the Foundation for Science and Technology, FCT (Portuguese Ministry of Science, Technology and Higher Education) (POCl-01-0145-FEDER-021177), under the project PTDC/SAU-EPI/121532/2010 and Unidade de Investigação em Epidemiologia, Instituto de Saúde Pública da Universidade do Porto Epidemiology Research Unit (EPIUnit) (POCl-01-0145-FEDER-006862; Ref. UID/DTP/04750/2013). SS received a grant from the Foundation for Science and Technology (SFRH/BD/81123/2011). AO receives

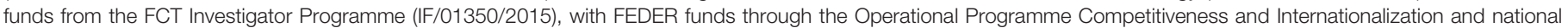
funding from the Foundation for Science and Technology, FCT, cofunded by the FCT and the POPH/FSE Program. The funders had no role in the design, analysis, or writing of this article.

Disclosure: The authors declared no conflict of interest.

Author contributions: SS and AO conceptualized and designed the study; SS and MS carried out the analyses; SS, CL, and AO wrote the paper. All authors read and approved the final version.

Additional Supporting Information may be found in the online version of this article.

Received: 8 December 2017; Accepted: 8 February 2018; Published online 25 March 2018. doi:10.1002/oby.22170 
TABLE 1 Characteristics of study participants $(n=2,531)$

\begin{tabular}{lc}
\hline & Mean (SD) \\
\hline Age, $\mathbf{y}$ & $6.7(0.49)$ \\
$\mathrm{BMI}, \mathrm{kg} / \mathrm{m}^{2}$ & $16.9(2.45)$ \\
BIA fat mass index, $\mathrm{kg} / \mathrm{m}^{2}$ & $3.1(2.27)$ \\
Waist-to-height ratio & $0.5(0.05)$ \\
Waist-to-hip ratio & $0.9(0.04)$ \\
Waist-to-thigh ratio & $1.6(0.10)$ \\
Waist-to-weight ratio, $\mathrm{cm} / \sqrt{ } \mathbf{k g}$ & $10.2(0.47)$ \\
DXA fat mass index, $\mathrm{kg} / \mathrm{m}^{4}$ & $3.7(1.32)$ \\
DXA central fat, $\mathrm{kg}$ & $3.3(1.71)$ \\
\end{tabular}

BIA, bioelectric impedance analysis; DXA, dual-energy $x$-ray absorptiometry.

A subsample of 2,531 children had whole-body dual-energy x-ray absorptiometry (DXA) measurements $(47.6 \%$ females) (details on nonresponse analyses given in Supporting Information Methods). The study was approved by the University of Porto Medical School S. João Hospital Centre ethics committee, and a signed informed consent according to the Declaration of Helsinki was obtained from all participants.

Anthropometrics were obtained by trained personnel with children in underwear and barefoot, according to standard procedures (5) (details given in Supporting Information Methods). BMI was calculated. Waist-to-height, waist-to-hip, and waist-to-thigh ratios were calculated as WC divided by height, hip, and thigh circumferences, respectively. The relationship between $\mathrm{WC}$ and weight was assessed by using a log-log regression analysis, which gave a slope of 0.5 , corresponding to the value by which weight should be raised in order to calculate a measure uncorrelated with it. Waist-to-weight ratio was calculated for the first time in the literature as $\mathrm{WC} /$ weight $^{0.5}$.

Tetrapolar bioelectric impedance analysis was performed (BIA 101 Anniversary; Akern, Florence, Italy). Fat-free mass was determined by using the Schaefer et al. equation, and fat mass was derived accordingly (6). Fat mass/height ${ }^{2}$ was calculated to obtain the fat

TABLE 2 Factor loadings obtained from principal component analysis for anthropometric and bioelectric impedance measures $(n=5,719)$

\begin{tabular}{lcc}
\hline & \multicolumn{2}{c}{ Factor loadings } \\
\cline { 2 - 3 } & Pattern 1 & Pattern 2 \\
\hline BMI, $\mathrm{kg} / \mathrm{m}^{2}$ & 0.98 & 0.05 \\
BIA fat mass index, $\mathrm{kg} / \mathrm{m}^{2}$ & 0.94 & 0.06 \\
Waits-to-height ratio & 0.87 & 0.45 \\
Waist-to-hip ratio & 0.28 & 0.88 \\
Waist-to-thigh ratio & -0.04 & 0.89 \\
Waist-to-weight ratio, cm/ $\sqrt{ } \mathrm{kg}$ & 0.20 & 0.90 \\
Variance explained & $45.3 \%$ & $43.0 \%$ \\
Cumulative variance explained & $45.3 \%$ & $88.3 \%$
\end{tabular}

BIA, bioelectric impedance analysis.
TABLE 3 Pearson's correlation coefficients of body fat patterns and single measures with DXA-derived measures in children $(n=2,531)$

\begin{tabular}{lcc}
\hline & $\begin{array}{c}\text { DXA fat mass } \\
\text { index, } \mathbf{k g} / \mathbf{m}^{4}\end{array}$ & $\begin{array}{c}\text { DXA central } \\
\text { fat, } \mathbf{k g}^{\mathbf{a}}\end{array}$ \\
\hline Pattern 1: fat quantity & $0.85(P<0.001)$ & $0(P=0.879)$ \\
Pattern 2: fat distribution & $0.10(P<0.001)$ & $0.35(P<0.001)$ \\
BMI, $\mathbf{k g} / \mathrm{m}^{2}$ & $0.83(P<0.001)$ & $0.08(P<0.001)$ \\
BIA fat mass index, $\mathbf{k g} / \mathrm{m}^{2}$ & $0.80(P<0.001)$ & $-0.02(P=0.305)$ \\
Waist-to-height ratio & $0.82(P<0.001)$ & $0.28(P<0.001)$ \\
Waist-to-hip ratio & $0.27(P<0.001)$ & $0.31(P<0.001)$ \\
Waist-to-thigh ratio & $0.02(P=0.462)$ & $0.30(P<0.001)$ \\
Waist-to-weight & $0.35(P<0.001)$ & $0.33(P<0.001)$ \\
$\quad$ ratio, $\mathbf{c m} / \sqrt{ } \mathbf{k g}$ & &
\end{tabular}

aAdjusted for total fat mass.

$\mathrm{BIA}$, bioelectric impedance analysis; DXA, dual-energy x-ray absorptiometry.

mass index (BIA-FMI), in which fat mass was effectively uncorrelated with height.

Whole-body DXA scans were performed (QDR 4500A; Hologic, Bedford, Massachusetts). Fat mass/height ${ }^{4}$ was calculated and was effectively uncorrelated with height. Central fat was assessed as trunk fat. Details on the comparison between fat mass obtained from BIA and DXA are given in Supporting Information Methods.

Principal component analysis was applied to BMI, waist-to-height, waist-to-hip, waist-to-thigh, and waist-to-weight ratios, and BIAFMI. Factors with eigenvalues $\geq 1.0$ were retained, and varimax rotation was performed. Factor loadings $>0.30$ were used in the interpretation of factors. The scores were calculated by using the regression method with standardized scores. Pearson's correlation coefficients of the scores obtained by principal component analysis and the single measures with DXA-FMI and central fat were obtained. Analyses were conducted by using R version 3.0.1 (The R Foundation, Vienna, Austria) and SPSS Statistics 21.0 (IBM Corp., Armonk, New York).

\section{Results}

The characteristics of participants are shown in Table 1. The following two independent body fat patterns, which explained $88.3 \%$ of total variance, were identified: a pattern 1, characterized by BMI, BIA-FMI, and waist-to-height ratio, and a pattern 2, characterized by waist-to-hip, waist-to-thigh, and waist-to-weight ratios (Table 2). Pattern 1 (fat quantity) presented a strong correlation with DXAFMI $(r=0.85 ; P<0.001)$, while pattern 2 (fat distribution) presented a moderate correlation with DXA central fat $(r=0.35$; $P<0.001$ ) (Table 3). The magnitude of the correlations with DXAderived measures was similar for body fat patterns and single measures. Waist-to-height ratio presented a strong correlation with DXAFMI $(r=0.82 ; P<0.001)$, while waist-to-thigh ratio presented a moderate correlation with DXA central fat $(r=0.30 ; P<0.001)$. Waist-to-hip and waist-to-weight ratios were similarly and moderately correlated with both DXA-derived measures. No sex 
differences were observed in the body fat patterns and in the associations investigated (data not shown).

\section{Discussion}

Pattern 1 combines well-known measures of total adiposity (BMI and BIA-FMI), and pattern 2 combines measures of regional adiposity (waist-to-hip and waist-to-thigh ratios); they seem to represent fat quantity and fat distribution, respectively. This is corroborated by the strong correlation between pattern 1 and DXA-FMI and the moderate correlation between pattern 2 and DXA central fat. Nonetheless, both body fat patterns showed similar correlations with DXA-derived measures as compared with single measures. Body fat patterns seem to add little value for estimating body fat in children. Fat distribution, using either patterns or single measures, seems to be more poorly measured than fat quantity.

Anthropometric indices based on WC may represent both fat quantity and distribution, which may raise controversial findings in population-based settings. In our study, waist-to-height, waist-to-hip, waist-to-thigh, and waist-to-weight ratios presented similar correlations with DXA central fat but great variability in the correlations with DXA-FMI. The waist-to-height ratio was strongly correlated with DXA-FMI but moderately correlated with DXA central fat. Because of the strong relation with fat quantity, this index seems unable to properly distinguish children according to their fat location and, thus, should be used as a proxy for total fat. On the other hand, waist-to-thigh ratio was not correlated with DXA-FMI and was moderately correlated with DXA central fat, and, thus, it seems to be a proxy for central fat. Waist-to-hip and waist-to-weight ratios were similarly and moderately correlated with both DXA-derived measures and, therefore, seem to be unable to distinguish between total and central fat. However, their correlations with DXA-FMI were much weaker compared with BMI, BIA-FMI, and waist-toheight ratio, suggesting that these indices are poor indicators of total fat. On the contrary, their correlations with DXA central fat were similar to waist-to-thigh ratio, showing the same ability to measure central fat, which is overall poorly measured by anthropometric indices. Therefore, among the anthropometric indices based on WC available in clinical practice, waist-to-hip and waist-to-weight ratios might be reasonable proxies for central fat. WC showed similar findings compared with waist-to-height ratio (data not shown).

Measurements of WC require adjustment for body height or weight if comparisons between individuals, or within individuals over time, are to be meaningful. Previous studies have shown that shorter subjects had a higher cardiometabolic risk than taller subjects with similar WC, which contributed to the definition of waist-to-height ratio as another proxy for central adiposity (7). The correction of WC for height allows for a single cutoff level to be used in different ethnic, age, and sex groups (8), while WC requires sex- and populationspecific cutoff levels to account for the effect of height on metabolic risk within different populations (9). However, adjustment of WC for height seems not enough to distinguish children according to their fat distribution. Two hypothetical children of identical height might have a different WC because of different body weights. Therefore, waist-to-height ratio remains influenced by body weight and, like WC, is a better index of total adiposity rather than of central adiposity, as corroborated by our findings. Previous studies have discussed whether WC adjusted for BMI is a better predictor of visceral fat in adults compared with WC and have reported contradictory results $(10,11)$. In our sample of 7 -year-old children, height explains $3.9 \%$ while weight explains $83.4 \%$ of the variance of $\mathrm{WC}$, and, thus, adjustment for weight seems to be appropriate in order to obtain an accurate measure of central adiposity. WC can be adjusted for weight as $\mathrm{WC} /$ weight $^{\mathrm{p}}$, in which $\mathrm{p}$ is the appropriate power by which to raise weight in order to obtain an index uncorrelated with it. Compared with waist-to-hip ratio and waist-to-thigh ratio, the other measures of fat distribution in our study, waist-to-weight ratio might be easier to obtain and less likely to have measurement error in most age groups. This was the first time waist-to-weight ratio as a proxy for central fat has been suggested; therefore, further studies are needed to address its predictive ability to identify children at risk of developing cardiometabolic disease.

Some strengths and limitations should be considered. The major strengths of this study were the population-based design with detailed childhood body fat measurements available. We relied on anthropometric measurements, which might have greater measurement error and be less accurate; however, they may be easier and cheaper to obtain in large epidemiological studies compared with imaging techniques of body composition (12). In this study, we also used DXA, which is an imaging technique that quantifies total and regional body fat content with high precision (12). Of the 5,719 children with anthropometrics and tetrapolar bioelectric impedance available, 2,531 children had DXA measurements. However, the nonresponse is unlikely to lead to biased effect estimates because children with and without DXA measurements did not differ regarding the sex, age, and anthropometric indices.

In conclusion, this study suggests that body fat patterns, compared with single anthropometric indices, add little value for estimating body fat quantity and distribution in children. Waist-to-height ratio seems to be a proxy for total fat (and not for central fat, as suggested in previous studies), while waist-to-hip, waist-to-thigh, and waist-to-weight ratios seem to be proxies for central fat. Waist-toweight ratio is a promising proxy for abdominal fat distribution, with population-based advantages over other measures, that warrants further research. $\mathrm{O}$

\section{Acknowledgments}

The article is also available online as part of a thesis (https://www.gene rationr.nl/wpcontent/uploads/2017/11/dissertation_Susana-Santos.pdf).

\section{(c) 2018 The Obesity Society}

\section{References}

1. Basterfield L, Pearce MS, Adamson AJ, et al. Effect of choice of outcome measure on studies of the etiology of obesity in children. Ann Epidemiol 2012;22:888-891.

2. Bouchard C. BMI, fat mass, abdominal adiposity and visceral fat: where is the 'beef'? Int J Obes 2007;31:1552-1553.

3. Katzmarzyk PT, Bouchard C. Where is the beef? Waist circumference is more highly correlated with BMI and total body fat than with abdominal visceral fat in children. Int J Obes 2014;38:753-754.

4. Larsen PS, Kamper-Jorgensen M, Adamson A, et al. Pregnancy and birth cohort resources in europe: a large opportunity for aetiological child health research. Paediatr Perinat Epidemiol 2013;27:393-414.

5. Gibson RS. Principles of Nutritional Assessment. 2nd ed. New York: Oxford University Press; 2005 
6. Schaefer F, Georgi M, Zieger A, et al. Usefulness of bioelectric impedance and skinfold measurements in predicting fat-free mass derived from total body potassium in children. Pediatr Res 1994;35:617-624.

7. Schneider HJ, Klotsche J, Silber S, et al. Measuring abdominal obesity: effects of height on distribution of cardiometabolic risk factors risk using waist circumference and waist-to-height ratio. Diabetes Care 2011;34:e7. doi: 10.2337/dc10-1794

8. Ashwell M, Hsieh SD. Six reasons why the waist-to-height ratio is a rapid and effective global indicator for health risks of obesity and how its use could simplify the international public health message on obesity. Int J Food Sci Nutr 2005;56:303-307.

9. Alberti KG, Eckel RH, Grundy SM, et al. Harmonizing the metabolic syndrome: a joint interim statement of the International Diabetes Federation Task Force on
Epidemiology and Prevention; National Heart, Lung, and Blood Institute; American Heart Association; World Heart Federation; International Atherosclerosis Society; and International Association for the Study of Obesity. Circulation 2009;120:1640-1645.

10. Berentzen TL, Angquist L, Kotronen A, et al. Waist circumference adjusted for body mass index and intra-abdominal fat mass. PLoS One 2012;7:e32213. doi: 10.1371/journal.pone.0032213

11. Janssen I, Heymsfield SB, Allison DB, et al. Body mass index and waist circumference independently contribute to the prediction of nonabdominal, abdominal subcutaneous, and visceral fat. Am J Clin Nutr 2002;75:683-688.

12. Wells JC, Fewtrell MS. Measuring body composition. Arch Dis Child 2006;91:612617. 